\title{
Mixed infection and clonal representativeness of a single sputum sample in tuberculosis patients from a penitentiary hospital in Georgia
}

\author{
Isdore C Shamputa*1,2, Levan Jugheli1,3, Nikoloz Sadradze ${ }^{3}$, Eve Willery ${ }^{4}$, \\ Françoise Portaels ${ }^{1}$, Philip Supply ${ }^{\dagger 4}$ and Leen Rigouts ${ }^{\dagger 1}$
}

\begin{abstract}
Address: ${ }^{1}$ Prince Leopold Institute of Tropical Medicine, Mycobacteriology Unit, Nationalestraat 155, B-2000 Antwerp, Belgium, ${ }^{2}$ Tropical Diseases Research Centre, Microbiology Unit, P. O. Box 71769, Ndola, Zambia, ${ }^{3}$ International Committee of the Red Cross, 4, Kedia Str. 380054, Tbilisi, Georgia and ${ }^{4}$ Laboratoire des Mécanismes Moléculaires de la Pathogenèse Bactérienne, INSERM U629, Institut de Biologie/Institut Pasteur de Lille, Lille, France

Email: Isdore C Shamputa* - icshamputa@itg.be; Levan Jugheli - levan_j@hotmail.com; Nikoloz Sadradze -nsadradze@yahoo.com; Eve Willery - eve.willery@ibl.fr; Françoise Portaels - portaels@itg.be; Philip Supply - philip.supply@ibl.fr; Leen Rigouts - lrigouts@itg.be

* Corresponding author †Equal contributors
\end{abstract}

Published: 17 July 2006

Respiratory Research 2006, 7:99 doi:10.1 186/1465-9921-7-99

This article is available from: http://respiratory-research.com/content/7/1/99

(c) 2006 Shamputa et al; licensee BioMed Central Ltd.

This is an Open Access article distributed under the terms of the Creative Commons Attribution License (http://creativecommons.org/licenses/by/2.0), which permits unrestricted use, distribution, and reproduction in any medium, provided the original work is properly cited.
Received: 05 April 2006

Accepted: 17 July 2006

\begin{abstract}
Background: Studies on recurrent tuberculosis (TB), TB molecular epidemiology and drug susceptibility testing rely on the analysis of one Mycobacterium tuberculosis isolate from a single sputum sample collected at different disease episodes. This scheme rests on the postulate that a culture of one sputum sample is homogeneous and representative of the total bacillary population in a patient.
\end{abstract}

Methods: We systematically analysed several pre-treatment isolates from each of 199 smearpositive male adult inmates admitted to a prison TB hospital by standard IS6//0 DNA fingerprinting, followed by PCR typing based on multiple loci containing variable number of tandem repeats (VNTRs) on a subset of isolates. Drug susceptibility testing (DST) was performed on all isolates for isoniazid, rifampicin, streptomycin and ethambutol.

Results: We found mixed infection in 26 (13.1\%) cases. In contrast, analysis of a single pretreatment isolate per patient would have led to missed mixed infections in all or 14 of these 26 cases by using only standard DNA fingerprinting or the PCR multilocus-based method, respectively. Differences in DST among isolates from the same patient were observed in 10 cases, of which 6 were from patients with mixed infection.

Conclusion: These results suggest that the actual heterogeneity of the bacillary population in patients, especially in high TB incidence settings, may be frequently underestimated using current analytical schemes. These findings have therefore important implications for correct interpretation and evaluation of molecular epidemiology data and in treatment evaluations.

\section{Background}

Tuberculosis (TB) has been traditionally assumed to result from a single infection with a single Mycobacterium tuberculosis strain, and this infection is thought to confer 
immunity to additional infections. Therefore, a recurrence of disease has been most often considered to be caused by endogenous reactivation of the strain that caused the original infection (relapse). Consequently, almost all current analytical schemes of clinical or research relevance are still based on examination of single isolates of given disease episodes, with implicit assumption that this isolate is representative of an homogeneous bacillary population.

This model of homogeneous infection has been revised by several studies using strain typing methods, which have demonstrated the occurrence of infection with clonally distinct strains, especially in high-incidence settings [1-3]. Both human immunodeficiency virus (HIV)-negative and HIV-positive individuals can be infected with more than one strain during a given disease episode (mixed infection), or re-infected by a second $M$. tuberculosis strain during a recurrent episode (exogenous re-infection). Such findings have important implications for control programs, vaccine development, evaluation of treatment regimens [4], and for epidemiological interpretation [3,5].

However, these studies of re-infection and mixed infection have so far been conducted by analysing the genotypes of the isolate from one sputum specimen from the initial and recurrent episodes or from one given episode, respectively [6-8]. Such approaches discount the old postulate that bacilli sequestered at different pulmonary infection sites are not necessarily released in the sample provided. Therefore, analysis of a single isolate might underestimate the actual heterogeneity of the bacillary population in the host. Conversely, the consequences of clonal heterogeneity on the representativeness of a single isolate have remained unknown hitherto.

Here, we have prospectively evaluated both the frequency of mixed infections and the clonal heterogeneity among clinical isolates from the same patient by analysing at least two pre-treatment isolates from each of 199 TB patients from a prison TB hospital in Georgia, consecutively enrolled over a period of three years. These isolates were analysed by using standard IS6110-restriction fragment length polymorphism (RFLP) genotyping as a first-line screening, followed by typing based on PCR amplification of 15 different loci containing mycobacterial interspersed repetitive unit-variable number of tandem repeats (MIRUVNTRs) for independent confirmation of simultaneous presence of multiple strains. The implications of the results for current analytical schemes of drug susceptibility testing (DST) and for evaluation of the contribution of re-infection to the epidemiology and pathogenesis of this disease are discussed.

\section{Methods \\ Study population}

All consecutive newly registered adult inmates ( $\geq 15$ years of age) with pulmonary TB admitted to a prison TB hospital near Tbilisi, Georgia from February 2001 to March 2004 were enrolled. All the TB patients included in our study were held in different detention centres and were only referred to the TB prison hospital after they were diagnosed with TB. TB history of study patients was according to the World Health Organization (WHO) guidelines [9]. TB notification rates in the general population during the study period was on average 117.3 cases per 100000 population (2001-2003) [10]. The HIV infection rate in the Georgian population is $<0.2 \%$ [11] and $1 \%$ among hospitalised TB patients [12]. Demographic data, including sex, age as well as date of diagnosis, clinical diagnosis, and treatment history, were obtained by review of medical and laboratory records. The study was approved by the Ministry of Justice of Georgia.

\section{Samples and cultures}

Three sputum samples were collected under strict supervision at the TB hospital from each of the patients within one week before the initiation of anti-TB treatment, which were collected as part of the routine patient investigation. Each sputum sample from all the patients studied was decontaminated by the modified Petroff's method [13] and cultured on Löwenstein-Jensen (L-J) medium at the National TB Reference Laboratory in Tbilisi, Georgia. The cultures were incubated at $37^{\circ} \mathrm{C}$ and read weekly for growth for a maximum period of 8 weeks. Identification of the primary isolates was done by classical methods.

\section{Drug-susceptibility testing}

DST was done on all M. tuberculosis isolates at the Prince Leopold Institute of Tropical Medicine (ITM) in Antwerp, Belgium by the proportion method on L-J medium containing $0.2 \mu \mathrm{g} / \mathrm{ml}$ isoniazid (INH), $40 \mu \mathrm{g} / \mathrm{ml}$ rifampicin (RIF), $4 \mu \mathrm{g} / \mathrm{ml}$ streptomycin (SM) and $2 \mu \mathrm{g} / \mathrm{ml}$ ethambutol (EMB) [14].

\section{DNA extraction}

DNA was extracted either by boiling bacterial suspensions for 5 min (MIRU-VNTR) or as previously described (RFLP and MIRU-VNTR) [15].

\section{DNA fingerprinting}

DNA fingerprinting of all M. tuberculosis isolates was performed by the IS6110-RFLP method [15]. Typing of isolates was done blinded i.e. only ITM culture numbers were used to identify isolates during DNA fingerprinting. Patient identities were only revealed when comparing the patterns (BioNumerics, version 3.0; Applied Maths, SintMartens-Latem, Belgium). DNA fingerprinting patterns from samples collected over the same period of time were 
reviewed to detect potential laboratory cross-contaminations or errors.

Secondary typing by MIRU-VNTR was performed on isolates from 30 patients with differences in their IS6110hybridisation patterns and on isolates from another 30 patients with identical IS6110-RFLP patterns (controls), using 15 MIRU-VNTR loci selected from a wider set of loci based on their variability in unrelated isolates and stability in clonally related isolates [16-21]. These loci have been found to be more discriminative and better for use in molecular epidemiology studies of TB (Supply et al., in preparation) than the 12 previously described loci [20], and they will be therefore proposed for standardisation. The number of repeats in the 15 target loci was determined after multiplex PCR with fluorescently labelled primers against regions flanking the repetitive sequences, electrophoretic separation and sizing of the PCR products using an ABI 3730 XL sequencer [2,19,22]. Specific precautions were taken to avoid and control cross contamination.

\section{Steps taken to minimise laboratory error}

To avoid swapping of sputum samples between patients, containers were pre-labelled with patient identities before sample collection. To minimise laboratory cross-contamination during decontamination and culture of samples, work was done in laminar flow cabinets, and only a limited number of specimens were processed at a time. In addition, study samples were received in batches both in Georgia and Belgium and were processed separately from all other samples received by the laboratory.

To minimise the risk of cross contamination during sample preparation for MIRU-VNTR typing, sample preparation for PCR, and the addition of DNA was done in a laminar flow cabinet. The H37Rv M. tuberculosis strain and water were included in each experiment as positive and negative controls, respectively. Reagent contamination could not be detected as all the negative controls were negative on amplification, and the correct number of repeats and no double alleles were detected from the positive controls.

\section{Statistical methods}

Analysis of variance (ANOVA) was used to compare continuous variables and the $\chi^{2}$ test was used for comparisons of proportions between test groups. The analyses were conducted using the Statistical Package for Social Sciences (SPSS version 14.0). A p value $<0.05$ was considered significant.

\section{Results \\ Mycobacterial cultures and patient characteristics}

Of the 385 eligible subjects, 186 patients were excluded because: only one sample was culture-positive for $M$. tuberculosis or yielded a good subculture upon receipt in Antwerp (102), only one isolate had IS6110-RFLP results (60), isolates were not available for IS6110-RFLP fingerprinting after shipment (23), and probable laboratory error (1). The conclusion on the latter case was based on the fact that one of the isolates from the latter patient had identical DNA fingerprinting patterns with an isolate from another patient processed on the same day. Thus, 199 patients with more than one $M$. tuberculosis isolate with IS6110-RFLP results were included in the analysis (Figure 1). All the study subjects were male, and the median age of the 198 patients with available age was 30 years (range, 20 to 63 years). Of these, 134 patients were new cases and the remaining 65 patients were retreatment cases. There was no statistically significant difference in the age ( $\mathrm{p}=$ $0.301)$ or retreatment types $(p=0.485)$ of patients that were excluded from the study and those that were included.

\section{Detection of mixed infections IS6 I I0-RFLP screening}

All sets of $M$. tuberculosis pre-treatment isolates available from 199 patients were genotyped by IS6110-RFLP. The number of IS6110 copies among these isolates ranged from 5 to 18 (median, 11 bands). Isolates from 169 patients showed identical patterns within each set, suggesting infection by a single strain. In contrast, isolates from 12 patients (patients 19-30) showed minor differences in IS6110-RFLP patterns within their respective sets ranging from 1 to 3 bands indicative of heterogeneous subpopulations, whereas isolates from 18 patients showed major differences of more than 3 bands suggestive of infection by multiple strains (mixed infection). Among the latter, isolates from 7 patients (patients 1-7) showed very distinct IS6110-RFLP patterns. Of the remaining 11 patients (patients 8-18), at least one isolate appeared to be a mixture of two different $M$. tuberculosis strains, as evidenced by possession of multiple overlapping bands compared to the other isolate(s) from the same patient (see Additional file 1, Table 2).

\section{MIRU-VNTR typing}

To study the observed heterogeneity further, all pre-treatment isolates from 30 patients with major (patients 1-18) and minor (patients 19-30) IS6110-RFLP differences were typed by MIRU-VNTR using 15 independent loci. In addition, all pre-treatment isolates from 30 patients with identical IS6110-RFLP patterns, presumably infected with a single strain, were also typed by MIRU-VNTR as controls. These control isolates were selected to cover the spectrum of IS6110 profiles among the different IS6110-RFLP clus- 


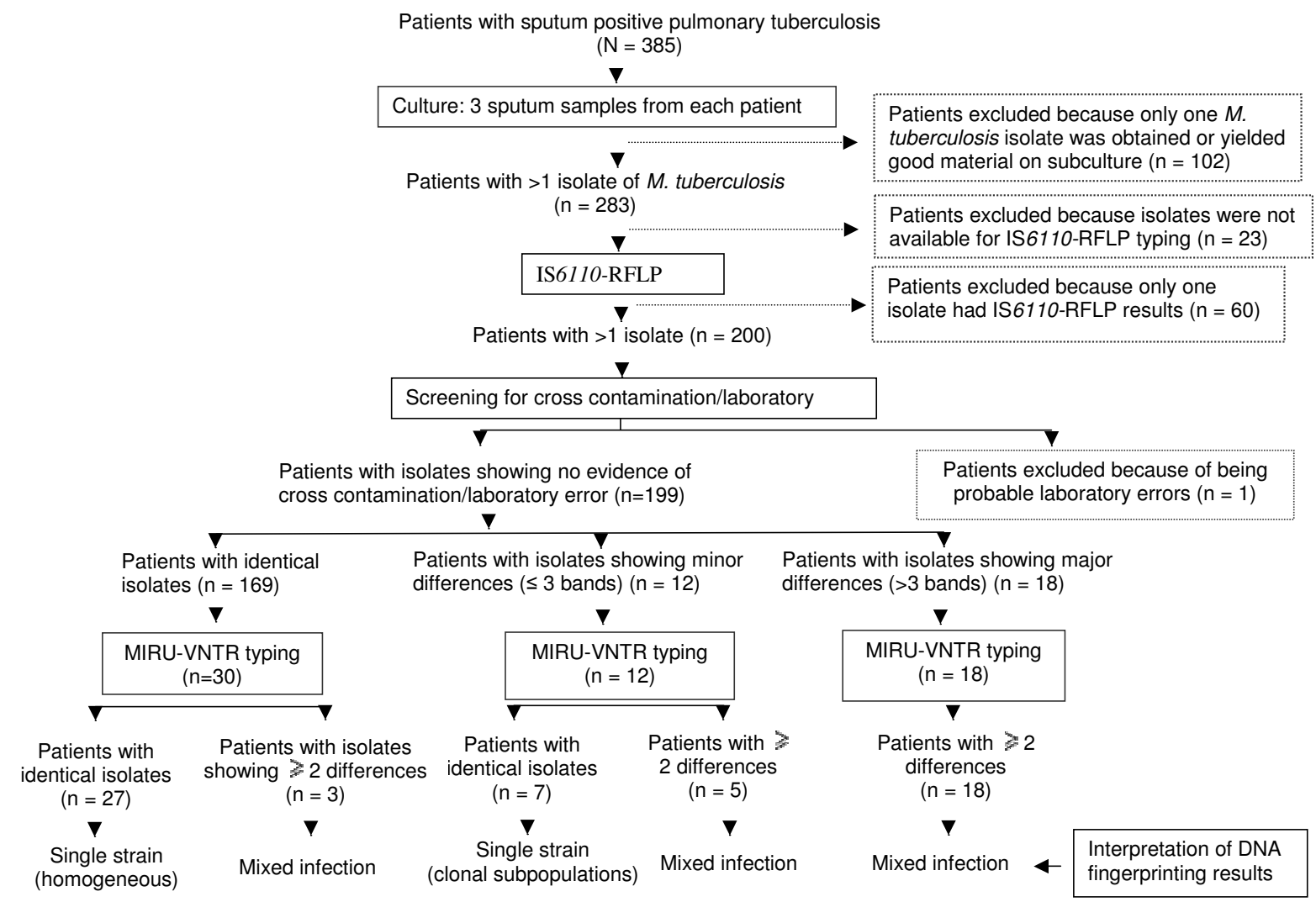

\section{Figure I}

Schematic diagram showing the grouping of patients according to culture results and subsequent IS6 I I0-RFLP and MIRUVNTR typing.

ters and unique isolates found among the 169 sets of isolates with conserved fingerprints within a set.

Consistently, respective isolates from the 18 patients with major IS6110 band differences were all found to have very distinct MIRU-VNTR profiles as well, corroborating the conclusion of mixed infection. More detailed analyses of the MIRU-VNTR results of these 18 patients showed that for some of them, different strains could be detected only in different sputum specimens, whereas for other patients the different strains were found to be simultaneously present within one sample. For example, isolates from two of these patients (patients 4 and 5) displayed MIRUVNTR profiles differing by 10 and 14 loci respectively, and all showed a single allele per locus. This observation provides evidence for the presence of a single strain per sample, but different from the strain from another specimen of the respective patient. Isolates from 14 other patients from this group (patients 1-3, 6-8, 11-18) showed a combination of single alleles differing among the respective pre-treatment samples, and double alleles detected in at least three loci (see Additional file 1, Table 2). One of the two alleles in these loci systematically corresponded to the single allele detected in the same locus of the other isolate(s) from the same patient, or the same double alleles were detected in both isolates. This finding indicates the simultaneous presence of at least two distinct strains, which can be detected in one or more sputa. Furthermore, even triple alleles were reproducibly amplified from three loci of both isolates from patient 10 , suggesting the simultaneous presence of three distinct strains. This conclusion was corroborated by the detection of double alleles at three other loci of one of the isolates, none of which was detected in the same locus of the other isolate from that patient. A similar phenomenon was also noticed for both isolates of patient 9, but without detection of any triple allele.

Similarly, the respective isolates from 5 of 12 patients with minor IS6110 band differences showed different and/or double alleles in at least three MIRU-VNTR loci, indicating mixed infections. Interestingly, mixed infections as 
Table I: Patient and drug susceptibility results of pre-treatment isolates from patients with variant DNA fingerprinting patterns

\begin{tabular}{|c|c|c|c|c|c|c|c|}
\hline \multirow[t]{2}{*}{ Patient no. } & \multirow[t]{2}{*}{ Retreatment type } & \multirow[t]{2}{*}{ Age (years) } & \multirow[t]{2}{*}{ Isolate no. } & \multicolumn{4}{|c|}{ Drug susceptibility testing } \\
\hline & & & & INH & RIF & SM & EMB \\
\hline \multicolumn{8}{|c|}{ Patients infected with multiple (mixed) M. tuberculosis strains } \\
\hline Patients with inc & aplete DST results & & & & & & \\
\hline \multirow[t]{2}{*}{2} & New case & 23 & a & NT & NT & NT & NT \\
\hline & & & $\mathrm{b}$ & $\mathrm{S}$ & $\mathrm{S}$ & $\mathrm{S}$ & $\mathrm{S}$ \\
\hline \multicolumn{8}{|c|}{ Patients with only pan-susceptible isolates } \\
\hline 6 & New case & 22 & $a+b+c$ & S & $\mathrm{S}$ & $\mathrm{S}$ & $\mathrm{S}$ \\
\hline 7 & New case & 36 & $a+b+c$ & S & S & $S$ & $\mathrm{~S}$ \\
\hline 8 & New case & 38 & $a+b$ & S & S & $S$ & $\mathrm{~S}$ \\
\hline 12 & New case & 41 & $a+b+c$ & S & S & S & S \\
\hline 17 & New case & 57 & $a+c$ & S & S & $\mathrm{S}$ & $\mathrm{S}$ \\
\hline 18 & New case & 34 & $a+c$ & S & S & $\mathrm{S}$ & $\mathrm{S}$ \\
\hline 19 & New case & 24 & $b+c$ & S & S & $S$ & $S$ \\
\hline 30 & New case & NA & $a+b+c$ & S & S & $S$ & $S$ \\
\hline \multicolumn{8}{|c|}{ Patients with identical drug resistant isolates } \\
\hline 5 & New case & 26 & $a+b$ & $\mathrm{R}$ & S & $\mathrm{S}$ & $\mathrm{S}$ \\
\hline 11 & New case & 29 & $a+b$ & S & S & $\mathrm{R}$ & $\mathrm{S}$ \\
\hline 10 & New case & 27 & $a+b+c$ & S & S & $\mathrm{R}$ & $\mathrm{S}$ \\
\hline 24 & New case & 58 & $b+c$ & S & S & $\mathrm{R}$ & $\mathrm{S}$ \\
\hline 33 & New case & 23 & $a+b$ & S & S & $\mathrm{R}$ & $\mathrm{S}$ \\
\hline 29 & Return after default & 29 & $a+b$ & S & S & $\mathrm{R}$ & $S$ \\
\hline 22 & Previously received non-official TB treatment & 27 & $a+b$ & S & S & $\mathrm{R}$ & $\mathrm{S}$ \\
\hline 16 & New case & 29 & $a+b$ & $\mathrm{R}$ & S & $\mathrm{R}$ & $\mathrm{S}$ \\
\hline 31 & Relapse case & 38 & $a+b$ & $\mathrm{R}$ & $\mathrm{R}$ & $\mathrm{R}$ & $\mathrm{R}$ \\
\hline 3 & New case & 44 & $a+b+c$ & R & $\mathrm{R}$ & $\mathrm{R}$ & $\mathrm{R}$ \\
\hline 32 & Failure case & 21 & $b+c$ & $\mathrm{R}$ & $\mathrm{R}$ & $\mathrm{R}$ & $\mathrm{R}$ \\
\hline \multicolumn{8}{|c|}{ Patients with different drug resistant isolates } \\
\hline \multirow[t]{2}{*}{14} & New case & 41 & $\mathrm{~b}$ & S & S & $\mathrm{S}$ & $\mathrm{S}$ \\
\hline & & & c & $\mathbf{R}$ & S & $\mathrm{S}$ & $\mathrm{S}$ \\
\hline \multirow[t]{2}{*}{13} & New case & 51 & $\mathrm{a}$ & $\mathrm{R}$ & S & $\mathrm{S}$ & $\mathrm{S}$ \\
\hline & & & c & $\mathrm{R}$ & S & $\mathbf{R}$ & $\mathrm{S}$ \\
\hline \multirow[t]{2}{*}{4} & New case & 36 & $\mathrm{a}$ & S & S & $\mathbf{S}$ & $\mathrm{S}$ \\
\hline & & & $b$ & S & S & $\mathbf{R}$ & $\mathrm{S}$ \\
\hline \multirow[t]{3}{*}{1} & Previously received non-official TB treatment & 50 & $\mathrm{a}$ & $\mathrm{S}$ & S & $\mathrm{R}$ & $\mathrm{S}$ \\
\hline & & & b & $\mathbf{R}$ & S & $\mathrm{R}$ & $\mathrm{S}$ \\
\hline & & & c & $\mathbf{R}$ & S & $\mathrm{R}$ & $S$ \\
\hline \multirow[t]{3}{*}{15} & Return after default & 29 & $\mathrm{a}$ & $\mathrm{R}$ & S & $S$ & $\mathrm{~S}$ \\
\hline & & & $b$ & $\mathrm{R}$ & S & $\mathbf{R}$ & $\mathrm{S}$ \\
\hline & & & c & $\mathrm{R}$ & S & $\mathbf{R}$ & $\mathrm{S}$ \\
\hline \multirow[t]{2}{*}{9} & New case & 24 & $\mathrm{a}$ & $\mathrm{R}$ & S & $\mathrm{R}$ & $S$ \\
\hline & & & c & $\mathbf{R}$ ? & $S$ & $\mathrm{R}$ & $\mathrm{S}$ \\
\hline
\end{tabular}

Patients infected with clonal subpopulations of the same $M$. tuberculosis strain Patients with only pan-susceptible isolates

$\begin{array}{lccccccc}25 & \text { New case } & 25 & b+c & S & S & S & S \\ 20 & \text { Relapse case } & 27 & a+b+c & S & S & S & S\end{array}$

Patients with identical drug resistant isolates

\begin{tabular}{|c|c|c|c|c|c|c|c|}
\hline 21 & New case & 43 & $b+c$ & $\mathrm{~S}$ & $\mathrm{~S}$ & $\mathrm{R}$ & $\mathrm{S}$ \\
\hline 26 & New case & 37 & $b+c$ & $\mathrm{~S}$ & $S$ & $\mathrm{R}$ & $\mathrm{S}$ \\
\hline 27 & New case & 27 & $a+b$ & $\mathrm{~S}$ & $S$ & $\mathrm{R}$ & $\mathrm{S}$ \\
\hline 28 & New case & 40 & $a+b+c$ & $\mathrm{~S}$ & $S$ & $\mathrm{R}$ & $\mathrm{S}$ \\
\hline 23 & New case & 32 & $a+b+c$ & $\mathrm{R}$ & $\mathrm{S}$ & $\mathrm{R}$ & $\mathrm{S}$ \\
\hline
\end{tabular}

Definitions of abbreviations: INH = isoniazid; RIF = rifampicin; $\mathrm{SM}=$ streptomycin; $\mathrm{EMB}=$ ethambutol, $\mathrm{S}=\mathrm{Susceptible}$; $\mathrm{R}=\mathrm{Resistant;} \mathrm{R}$ ? = borderline resistance; NT = not tested; $a=$ first pretreatment sputum; $b=$ second pretreatment sputum; $c=$ third pretreatment sputum; NA = not available. 
Table 2: Results of 33 patients with multiple pre-treatment isolates showing variant DNA fingerprinting patterns

\begin{tabular}{|c|c|c|c|}
\hline Patient no. & No. of bands different by IS6 I I 0-RFLP & No. of loci with different/double alleles & Interpretation \\
\hline I & $>3$ & 10 & Mixed infection \\
\hline 2 & $>3$ & 10 & Mixed infection \\
\hline 3 & $>3$ & 12 & Mixed infection \\
\hline 4 & $>3$ & 14 & Mixed infection \\
\hline 5 & $>3$ & 10 & Mixed infection \\
\hline 6 & $>3$ & 11 & Mixed infection \\
\hline 7 & $>3$ & 13 & Mixed infection \\
\hline 8 & $>3$ & 8 & Mixed infection \\
\hline 9 & $>3$ & 11 & Mixed infection \\
\hline 10 & $>3$ & 15 & Mixed infection \\
\hline 11 & $>3$ & 10 & Mixed infection \\
\hline 12 & $>3$ & 12 & Mixed infection \\
\hline 13 & $>3$ & 8 & Mixed infection \\
\hline 14 & $>3$ & 7 & Mixed infection \\
\hline 15 & $>3$ & 9 & Mixed infection \\
\hline 16 & $>3$ & 10 & Mixed infection \\
\hline 17 & $>3$ & 9 & Mixed infection \\
\hline 18 & $>3$ & 12 & Mixed infection \\
\hline 19 & 1 & 5 & Mixed infection \\
\hline 29 & I & 4 & Mixed infection \\
\hline 30 & 3 & 7 & Mixed infection \\
\hline 31 & 0 & 2 & Mixed infection \\
\hline 32 & 0 & 7 & Mixed infection \\
\hline 33 & 0 & 10 & Mixed infection \\
\hline 22 & I & 4 & Mixed infection \\
\hline 24 & I & 13 & Mixed infection \\
\hline 20 & I & 0 & Identical/subpopulations \\
\hline 21 & i & 0 & Identical/subpopulations \\
\hline 23 & I & 0 & Identical/subpopulations \\
\hline 25 & I & 0 & Identical/subpopulations \\
\hline 26 & i & 0 & Identical/subpopulations \\
\hline 27 & I & 0 & Identical/subpopulations \\
\hline 28 & 3 & 0 & Identical/subpopulations \\
\hline
\end{tabular}

Patients are arranged according to the interpretation

defined on the same MIRU-VNTR basis were also revealed among 3 of the 30 patients whose isolates had identical IS6110 fingerprints. Detection of mixed infections by MIRU-VNTR typing among patients with isolates showing identical IS6110-RFLP patterns is not surprising because the former method includes an amplification step and a more sensitive, fluorescence-based, detection system.

Identical MIRU-VNTR profiles were obtained among the isolates from the 7 remaining patients with minor IS6110 band differences (mostly one band, at most 3 bands), and from the 27 remaining control sets with conserved IS6110 fingerprints. In keeping with previous studies $[2,23,24]$, the former group was conservatively assigned to IS6110 clonal variants from one original infecting strain and were thus defined as clonal subpopulations, while the latter group corresponded to cases of infection by a single strain, as defined by both typing methods used.
Based on IS6110-RFLP data on all isolates and MIRUVNTR data on isolates from 60 patients we found infection with a single M. tuberculosis strain either homogeneous or with some clonal subpopulation in $173(86.9 \%)$ of 199 patients, and mixed infection with two or three distinct strains in $26(13.1 \%)$ patients. Crucially, using the above rules for identification of mixed infections (detection of double alleles in multiple loci), analysis of a single pre-treatment isolate per patient would have led to missed mixed infection in 14 of these cases using MIRU-VNTR typing. Analysis of a single pre-treatment isolate per patient would have even led to missed mixed infection in all cases using standard IS6110-RFLP, as no evidence of strain mixture could be detected in single patterns.

Twenty-one of the 26 patients with mixed infection were new TB cases, 1 was a treatment failure case, 3 returned after default while 1 had previously received unofficial TB treatment (Table 1). The distribution of the treatment history of TB among these cases was not significantly differ- 
ent from the distribution among those infected with a single homogeneous or heterogeneous strain $\left(\chi^{2}, \mathrm{p}=\right.$ 0.117).

\section{Drug susceptibility testing}

Phenotypic DST classified 80 of 199 patients as having been infected with pan-susceptible isolates and 20 patients with multi-drug resistant (MDR) isolates. The remaining 99 patients were infected with isolates that were resistant to at least one drug, but not MDR.

From 25 out of 26 mixed infection cases with known DST results for all isolates, 8 patients were infected with only pan-susceptible strains, while the other cases showed resistance in at least one of the isolate. Out of these, clear differences in resistance patterns among isolates from the same patient were observed only in 5 cases (patients 1, 4, 13, 14, 15, Table 1). In another case (patient 9) however, one isolate showed a clear resistance to INH whereas a borderline result was obtained in the other. Isolates from patients 1 and 14 differed in their susceptibility to INH, which was confirmed by sequencing of the kat $\mathrm{G}$ and inh $\mathrm{A}$ genes (data not shown). The resistant isolate for patient 9 was confirmed by sequencing i.e. presence of a novel $T \rightarrow C$ (in contrast to the previously reported $\mathrm{T} \rightarrow \mathrm{A}$ or $\mathrm{G}$ ) mutation at position -8 of the inhA gene [24]. Interestingly, a mixture of both a wild type $(\mathrm{T})$ and the novel mutation (C) was obtained at the same position for the isolate with a borderline result, thereby corroborating our MIRUVNTR findings. Patient 9 and 14 were new cases, while patient 1 had previously received unofficial anti-TB treatment. Difference in SM resistance was observed in the remaining 3 patients $(4,13,15)$, but this was not investigated further.

In contrast to the above mixed infection cases with isolates showing different DST patterns, it is remarkable to note that the remaining 11 patients were infected with two or three different $M$. tuberculosis strains that showed exactly the same DST pattern: 1 mono-INH-resistant, 6 mono-SM-resistant, 2 INH + SM-resistant and 3 MDR. Eight of these patients were classified as new cases (Table $1)$.

Finally, among the 7 patients with clonal subpopulations, none showed differences in DST among their respective sets of isolates whereas among the patients with genetically homogeneous bacterial populations, one retreatment and three new cases showed a difference in SM among the isolates (data not shown).

\section{Discussion}

This report simultaneously assessed the validity of two interdependent postulates on which standard analytical schemes rely: (i) that a TB patient can only be infected with a single homogeneous $M$. tuberculosis strain at any given time, and (ii) that an isolate from a single sputum specimen is representative of the total bacillary population in a patient. Therefore, we systematically compared the genetic relatedness of $M$. tuberculosis isolates from multiple sputum samples collected prior to the initiation of anti-TB therapy from each of 199 smear-positive inmates admitted to a prison TB hospital. By using two independent genotyping methods to differentiate strains, we detected infection with two or even three distinct $M$. tuberculosis strains in $13.1 \%$ of the samples analysed. There was no significant difference in the proportion of retreatment cases between the excluded and included patients.

The mixed infection rate observed in this prison population can not be extrapolated to the general population because of overcrowding and higher incidence of TB in the prisons compared to the general population $(5,995 / 100$ 000 vs. 155/100 000 population, respectively) [26]. However, we believe that the so called "cheating" (i.e. prisoners attempt to submit sputa mixed with that of other prisoners suspected of having smear-positive TB, so that they can be diagnosed with $\mathrm{TB}$ and transferred to the prison $\mathrm{TB}$ hospital with better living conditions than in other detention centres) had a low influence on our estimation of this phenomenon, if any, due to the strict and active surveillance by an aware staff at the sputum collection step inside the TB hospital. Likewise, laboratory cross-contamination is an unlikely explanation for the high frequency of mixed infection detected because of the specific precautions taken.

The use of IS6110-RFLP-typing as an initial screening method may have led to some underestimation of mixed infection because this method has inherent limitations to detect mixed infections within a single isolate since various bands in a given profile can represent one or more strains. Moreover, it remains unclear to what extent low ratios of one of the strains present in a mixture are reflected in low-intensity bands [27] or not detected at all. The latter is evidenced by the detection of mixed infections by MIRU-VNTR among 3 (10\%) of 30 patients with isolates that showed identical IS6110-RFLP patterns but double alleles in multiple loci within one isolate by MIRU-VNTR. By extrapolation, this suggests that up to 14 (7.0\%) mixed infections might have been additionally detected among the other 139 patients with isolates that had identical IS6110-RFLP patterns if they were also tested by MIRU-VNTR. There was no statistically significant difference in retreatment type between new and previously treated cases among the patients whose isolates were only typed by IS6110-RFLP and those whose isolates were additionally typed by MIRU-VNTR $\left(\chi^{2}, \mathrm{p}=0.15\right)$. Finally, some mixed infections could have remained undetected 
by MIRU-VNTR typing itself although this PCR-based method is able to detect ratios of a given strain as low as 1:99 [28].

Detection of genetically distinct strains among multiple pre-treatment sputum samples, as well as within a single sputum specimen might reflect separate lesions in the lungs containing different $M$. tuberculosis strains and opening simultaneously or consecutively as also suggested from a previous study [29]. Regardless of the explanation, it is crucial to note that if only the first pretreatment sample was analysed by standard IS6110 fingerprinting or by MIRU-VNTR typing, none or only about half of the mixed infection cases detected by analysis of multiple pre-treatment samples (14 cases vs. 26 cases) would have been identified. These observations imply that analysis of a single isolate, especially in high incidence settings may underestimate the actual heterogeneity of the bacillary population in the host.

It is relevant to observe that the $13.1 \%$ of mixed infections detected in this prison population is relatively close to the frequency of $19 \%$ recently reported in the study of Warren et al. in a general population of a setting with an incidence $1000 / 100000$ population [3]. Although the two values are not directly comparable as this latter evaluation was limited to the detection of patients simultaneously infected with strains of both the Beijing and non-Beijing lineages, we predict that their value is likely an underestimation as only single isolates per patient were analysed in that case. Similarly, previous studies in high incidence settings might have overestimated the contribution of reinfection vs. relapse due to undetected initial mixed infection $[7,29]$. Our observations imply that for specific research studies analyses of several isolates from different sputum samples at each disease episode (before and after treatment), especially in high TB incidence settings might be helpful in distinguishing true reinfection vs. relapse and/or mixed infection, preferably using a PCR-based typing method like MIRU-VNTR.

From the 26 proven mixed infection cases in our study, $30 \%$ harboured only pan-susceptible strains, whereas $70 \%$ showed any resistance in at least one of the isolates. In only 6 cases was mixed infection reflected in a variant DST profile. Remarkably, the remaining patients were infected with two or three strains seemingly showing identical resistance profiles. Although most of these patients were new cases according to WHO definitions [9], we can not completely exclude the possibility that they might have taken TB drugs for less than one month, and therefore both strains might have acquired resistance as a result of the same drug pressure. On the other hand, independent infection with two or three strains showing exactly the same resistance profile for each of so many patients is very doubtful as well, even in a setting with a high rate of drug-resistant TB. Most probably, such frequent observations of identical resistance profiles among respective isolates from these mixed infections reflect the systematic presence of both a susceptible and a resistant strain in the corresponding specimens. This systematic duality was evidenced by the systematic detection of double alleles in the MIRU-VNTR patterns in the isolates from all these cases but one. In such conditions, the simultaneous growth of a (more) resistant strain will mask that of susceptible (or partly resistant) strains in DST assays either completely or partly resulting in either resistant isolates or isolates with borderline results (patient 9). In such situations, culturing and DST of single pre-treatment sputum had generally no predictable adverse consequences for the appropriateness of the treatment regimen of the respective patients.

As mentioned above, variant DST profiles were detected as a result of mixed infections in only $6(3.0 \%)$ of the 199 patients. This finding lends support to previous reports that initial mixed infections may actually be responsible for changes in DST patterns in isolates of some patients [29-31].

In general, our findings suggest that single-isolate analyses can be used for routine DST in most settings, except for some high drug resistant-TB-prevalent settings. However, for specific research studies like treatment evaluation and clinical trials, testing multiple isolates from different sputum samples at each disease episode could help in determining the respective contribution of mixed infection and reinfection versus relapse with gradual development of drug resistance, especially by PCR-based typing methods such as MIRU-VNTR.

Although the high rates of mixed infection in this prison setting can not be extrapolated to the general population with a lower risk of TB transmission, these findings nonetheless indicate that an initial infection is unable to provide protection against a subsequent infection in these populations, which have implications for the development and trials of new vaccines [3]. Because higher rates of mixed infection imply possible higher rates of super infection, the protective effect of an initial infection against a subsequent infection may be even lower than expected. This parameter needs to be taken into account in the development of new prophylactic approaches.

\section{Conclusion}

This study has demonstrated that different pre-treatment sputum samples from a patient can harbour distinct $M$. tuberculosis strains. In addition, the study has shown the occurrence of varying DST patterns among multiple pretreatment isolates, which might indicate mixed infection 
or ongoing acquisition of drug resistance. Our findings are important for the correct interpretation of molecular epidemiology data in follow-up studies in high incidence settings and in treatment evaluations.

\section{Competing interests}

The author(s) declare that they have no competing interests.

\section{Authors' contributions}

ICS: Performed DNA fingerprinting, evaluated the data, drafted and reviewed the manuscript. LJ: Co-ordinated the study and helped in drafting the manuscript. NS: Supervised sample collection, culture and identification of mycobacteria. EW: Participated in MIRU-VNTR typing. FP: Conceived of the study, participated in its design and reviewed the manuscript. PS: Supervised MIRU-VNTR typing, evaluated the data, helped in drafting and revising the manuscript. LR: Conceived of the study, participated in its design, co-ordination and evaluation of data, and helped in drafting the manuscript. All authors read and approved the final manuscript.

\section{Additional material}

\section{Additional File 1}

DNA fingerprinting results of pre-treatment $\mathrm{M}$. tuberculosis isolates with variant patterns from each of the respective 33 patients. $a=$ first pretreatment sample; $b=$ second pre-treatment sample; $c=$ third pre-treatment isolate from each patient; $d=M I R U-V N T R$ loci are listed according to their position (in kilobases) on the H37RV genome. Alternative designations are indicated in parentheses. For isolates with minor IS6110RFLP variations, arrows indicate additional band $(s) ; N D=$ not determined; *A third allele was detected at the respective locus. Al1, Al2 = Allele 1 and Allele 2, respectively. $2 S$ or $3 S=$ variant alleles in locus MIRU 04, similar to those in the H37RV and BCG genomes [20]. Click here for file

[http://www.biomedcentral.com/content/supplementary/14659921-7-99-S1.jpeg]

\section{Acknowledgements}

This study was funded by the Belgische Nationale Bond Tegen de Tuberculose (BNBTTB) and the International Committee of the Red Cross (ICRC), and was also partially supported by the Fund for Scientific Research of Flanders (Brussels, Belgium, grant no. G.047I.03N) and the Damien Foundation (Brussels, Belgium). I.C.S. acknowledges a scholarship from Ackerman \& van Haaren. We express special thanks to the staff of the National Tuberculosis Reference Laboratory of Georgia for their outstanding work. We are grateful to the Prison tuberculosis Colony staff of Georgia and the technical staff of the Institute of Tropical Medicine for their excellent assistance. We are indebted to the staff of the Ministry of Justice of Georgia for granting permission to conduct the study. We thank Vincent Vatin (CNRS UMR 8090, Institut de Biologie/Institut Pasteur de Lille) for providing laboratory facilities and Anne Buvè (ITM Antwerp) for her help with statistics. P.S. is a researcher of the Centre National de la Recherche Scientifique (CNRS, France).

\section{References}

I. Das S, Narayanan S, Hari L, Mohan NS, Somasundaram S, Selvakumar $\mathrm{N}$, Narayanan P: Simultaneous infection with multiple strains of Mycobacterium tuberculosis identified by restriction fragment length polymorphism analysis. Int J Tuberc Lung Dis 2004, 8:267-270.

2. Shamputa IC, Rigouts L, Eyongeta LA, El Aila NA, van Deun A, Salim AH, Willery E, Locht C, Supply P, Portaels F: Genotypic and phenotypic heterogeneity among Mycobacterium tuberculosis isolates from pulmonary tuberculosis patients. J Clin Microbiol 2002, 42:5528-5536.

3. Warren RM, Victor TC, Streicher EM, Richardson M, Beyers N, van Pittius NC, van Helden PD: Patients with active tuberculosis often have different strains in the same sputum specimen. Am J Respir Crit Care Med 2004, 169:610-6I4.

4. Fine PE, Small PM: Exogenous reinfection in tuberculosis. N Eng J Med 1999, 34I:I226-I227.

5. Richardson M, Carroll NM, van Der Spuy GD, Salker F, Munch Z, Gie RP, Warren RM, Beyers N, van Helden PD: Multiple Mycobacterium tuberculosis strains in early cultures from patients in a high-incidence community setting. J Clin Microbiol 2002, 40:2750-2754.

6. Jasmer RM, Bozeman L, Schwartzman K, Cave MD, Saukkonen JJ, Metchock B, Khan A, Burman WJ, Tuberculosis Trials Consortium: Recurrent tuberculosis in the United States and Cananda Relapse or reinfection? Am J Respir Crit Care Med 2004, I 70:1360-1336.

7. Sonnenberg P, Murray J, Glynn JR, Shearer S, Kambashi B, GodfreyFaussett $P$ : HIV-I and recurrence, relapse, and reinfection of tuberculosis after cure: a cohort study in South African mineworkers. Lancet 200I, 358:1687-1693.

8. Sonnenberg P, Murray J, Shearer S, Glynn JR, Kambashi B, GodfreyFaussett $P$ : Tuberculosis treatment failure and drug resistance - same strain or reinfection? Trans R Soc Trop Med Hyg 2000, 94:603-607.

9. World Health Organization: Guidelines for surveillance of drug resistance in tuberculosis. Geneva, Switzerland. WHO/TB/ 2003.320.

10. EuroTB and the national coordinators for tuberculosis surveillance in the WHO European Region: Surveillance of tuberculosis in Europe. Report on tuberculosis cases notified in 2003 Institut de veille sanitaire, Saint-Maurice, France; 2005.

II. UNAIDS: Report on the global HIVIAIDS epidemic: 4th global report 2004.

12. Richards DC, Mikiashvili T, Parris JJ, Kourbatova EV, Wilson JCE, SHubladze N, Tsertvadze T, Khechinashvili G, del Rio C, Blumberg HM: High prevalence of hepatitis $C$ virus but not HIV coinfection among patients with tuberculosis in Georgia. Int J Tuberc Lung Dis 2006, 1 0:396-40I.

13. Kent PT, Kubica PG: Public health bacteriology, a guide to the level III laboratory Centers for Disease Control, Atlanta, Ga; 1985.

14. Canetti G, Fox W, Khomenko A, Mahler HT, Menon NK, Mitchison DA, Rist N, Smelev NA: Advances in techniques of testing mycobacterial drug sensitivity and the use of sensitivity tests in tuberculosis control programs. Bull WHO 1969, 4I:2I-43.

15. van Embden JD, Cave MD, Crawford JT, Dale JW, Eisenach KD, Gicquel B, Hermans P, Martin C, McAdam R, Shinnick TM, Small PM: Strain identification of Mycobacterium tuberculosis by DNA fingerprinting: recommendations for a standardized methodology. J Clin Microbiol 1993, 3 I:406-409.

16. Frothingham R, Meeker-O'Connell WA: Genetic diversity in the Mycobacterium tuberculosis complex based on variable numbers of tandem DNA repeats. Microbiology 1998, I 44: I I89-I I 96.

17. Le Fleche P, Fabre M, Denoeud F, Koeck JL, Vergnaud G: High resolution, on-line identification of strains from the Mycobacterium tuberculosis complex based on tandem repeat typing. BMC Microbiol 2002, 2:37.

18. Roring S, Scott A, Brittain D, Walker I, Hewinson G, Neil S, Skuce R: Development of variable-number tandem repeat typing of Mycobacterium bovis: comparison of results with those obtained by using existing exact tandem repeats and spoligotyping. J Clin Microbiol 2002, 40:21 26-2133.

19. Supply P, Lesjean S, Savine E, Kremer K, van Soolingen D, Locht C: Automated high-throughput genotyping for study of globalepidemiology of Mycobacterium tuberculosis based on mycobacterial interspersed repetitive units. J Clin Microbiol 200I, 39:3563-357l. 
20. Supply P, Mazars E, Lesjean S, Vincent V, Gicquel B, Locht C: Variable human minisatellite-like regions in the Mycobacterium tuberculosis genome. Mol Microbiol 2000, 36:762-771.

21. Warren RM, Victor TC, Streicher EM, Richardson M, van der Spuy GD, Johnson R, Chihota VN, Locht C, Supply P, van Helden PD: Clonal expansion of a globally disseminated lineage of Mycobacterium tuberculosis with low IS6 II 0 copy numbers. J Clin Microbiol 2004, 42:5774-5782.

22. Allix C, Supply P, Fauville-Dufaux M: Utility of fast mycobacterial interspersed repetitive unit-variable number tandem repeat genotyping in clinical mycobacteriological analysis. Clin Infect Dis 2004, 39:783-789.

23. Cave MD, Eisenach KD, Templeton G, Salfinger M, Mazurek G, Bates $\mathrm{JH}$, Crawford JT: Stability of DNA fingerprinting patterns produced with IS6IIO in strains of Mycobacterium tuberculosis. J Clin Microbiol 1994, 32:262-266.

24. Niemann S, Richter E, Rüsch-Gerdes S: Stability of Mycobacterium tuberculosis IS6IIO restriction fragment length polymorphism patterns and spoligotypes determined by analyzing serial isolates from patients with drug-resistant tuberculosis. J Clin Microbiol 1999, 37:409-4I2.

25. Ramaswamy S, Musser JM: Molecular genetic basis of antimicrobial agent resistance in Mycobacterium tuberculosis: 1998 update. Tuber Lung Dis 1998, 79:3-29.

26. World Health Organization: Tuberculosis control in prisons. Geneva, Switzerland. WHO/CDS/TB/2000.28I .

27. De Boer AS, Kremer K, Borgdorff MW, De Haas PE, Heersma HF, van Soolingen D: Genetic heterogeneity in Mycobacterium tuberculosis isolates reflected in IS 6110 restriction fragment length polymorphism patterns as low-intensity bands. J Clin Microbiol 2000, 38:4478-4484.

28. Garcia de Viedma D, Alonso Rodriguez N, Andres S, Ruiz Serrano MJ, Bouza $E$ : Characterization of clonal complexity in tuberculosis by mycobacterial interspersed repetitive unit-variablenumber tandem repeat typing. I Clin Microbiol 2005, 43:5660-5664.

29. Braden CR, Morlock GP, Woodley CL, Johnson KR, Colombel AC Cave MD, Yang Z, Valway SE, Onorato IM, Crawford JT: Simultaneous infection with multiple strains of Mycobacterium tuberculosis. Clin Infect Dis 200I, 33:e42-e47.

30. van Rie A, Victor TC, Richardson M, Johnson R, van der Spuy GD, Murray EJ, Beyers N, van Pittius NCG, van Helden PD, Warren RM Reinfection and Mixed Infection Cause Changing Mycobacterium tuberculosis Drug-Resistance Patterns. Am J Respir Crit Care Med 2005, 172:636-642.

31. Baldeviano-Vidalon GC, Quispe-Torres N, Bonilla-Asalde C, Gastiaburu-Rodriguez D, Pro-Cuba JE, Llanos-Zavalaga F: Multiple infection with resistant and sensitive $M$. tuberculosis strains during treatment of pulmonary tuberculosis patients. Int J Tuberc Lung Dis 2005, 9:1 I55-1160.
Publish with Biomed Central and every scientist can read your work free of charge

"BioMed Central will be the most significant development for disseminating the results of biomedical research in our lifetime. "

Sir Paul Nurse, Cancer Research UK

Your research papers will be:

- available free of charge to the entire biomedical community

- peer reviewed and published immediately upon acceptance

- cited in PubMed and archived on PubMed Central

- yours - you keep the copyright

Submit your manuscript here:

http://www.biomedcentral.com/info/publishing_adv.asp
BiolMedcentral 